$\underline{\underline{\text { IIIIIIII }}}$ Open Access Articles

\title{
2D-material-enabled multifunctional mid-IR optoelectronics
}

The MIT Faculty has made this article openly available. Please share how this access benefits you. Your story matters.

\begin{tabular}{|l|l|}
\hline Citation & $\begin{array}{l}\text { Deckoff-Jones, Skylar, Wang, Yixiu, Lin, Hongtao, Wu, Wenzhuo and } \\
\text { Hu, Juejun. 2020. "2D-material-enabled multifunctional mid-IR } \\
\text { optoelectronics." Proceedings of SPIE - The International Society for } \\
\text { Optical Engineering, 11284. }\end{array}$ \\
\hline As Published & $10.1117 / 12.2543619$ \\
\hline Publisher & SPIE \\
\hline \hline Version & Final published version \\
\hline Citable link & https://hdl.handle.net/1721.1/136710 \\
\hline Terms of Use & $\begin{array}{l}\text { Article is made available in accordance with the publisher's } \\
\text { policy and may be subject to US copyright law. Please refer to the } \\
\text { publisher's site for terms of use. }\end{array}$ \\
\hline
\end{tabular}




\section{D-material-enabled multifunctional mid-IR optoelectronics}

Deckoff-Jones, Skylar, Wang, Yixiu, Lin, Hongtao, Wu, Wenzhuo, Hu, Juejun

Skylar Deckoff-Jones, Yixiu Wang, Hongtao Lin, Wenzhuo Wu, Juejun Hu, "2D-material-enabled multifunctional mid-IR optoelectronics," Proc. SPIE 11284, Smart Photonic and Optoelectronic Integrated Circuits XXII, $112841 \mathrm{U}$ (26 February 2020); doi: 10.1117/12.2543619

SPIE. Event: SPIE OPTO, 2020, San Francisco, California, United States 


\title{
2D-material-enabled multifunctional mid-IR optoelectronics
}

\author{
Skylar Deckoff-Jones ${ }^{\mathrm{a}}$, Yixiu Wang ${ }^{\mathrm{b}}$, Hongtao Lin ${ }^{\mathrm{a}}$, Wenzhuo $\mathrm{Wu}^{\mathrm{b}}$, Juejun $\mathrm{Hu}^{* \mathrm{a}}$ \\ ${ }^{a}$ Department of Materials Science and Engineering, Massachusetts Institute of Technology, \\ Cambridge, Massachusetts 02139, USA; ${ }^{\mathrm{b} S}$ School of Industrial Engineering, Purdue University, West \\ Lafayette, Indiana, United States
}

\begin{abstract}
New narrow-gap two-dimensional (2-D) semiconductors exemplified by black phosphorus and tellurene are promising material candidates for mid-IR optoelectronic devices. In particular, tellurene, atomically thin crystals of elemental tellurium, is an emerging narrow-gap 2-D semiconductor amenable to scalable solution-based synthesis and large-area deposition. It uniquely combines tunable bandgap energies, high carrier mobility, exceptionally large electro-optic activity, and superior chemical stability, making it a promising and versatile material platform for mid-infrared photonics. Here we discuss the design and experimental realization of integrated photonic devices based on tellurene and other 2-D semiconductors specifically for the mid-IR spectral regime based on a chalcogenide glass (ChG) photonic platform.
\end{abstract}

Keywords: 2D materials, mid-infrared, detector, modulator, semiconductor

\section{INTRODUCTION}

Chalcogenide glasses have proven themselves a valuable material for integrated photonics applications due to their high refractive index, broadband transparency, and versatile fabrication conditions ${ }^{1}$. The family of glasses receives its name from its composition of chalcogen atoms - S, Se, and Te. Relative compositions can be tuned to control the band edge, mechanical properties, and refractive index ( $n$ from 2 to 3.5$)^{2,3}$. ChGs' high refractive index enables waveguides with strong mode confinement, allowing extremely low-loss, small-footprint photonic circuits ${ }^{4-7}$. Furthermore, their broad transparency window extends from visible to the far-IR, making them ideal optical media for mid-IR $(2-20 \mu \mathrm{m})$ photonics $^{8,9}$. Previously we have demonstrated ChG based sensors, filters, and active devices ${ }^{10-12}$. ChGs have also demonstrated photosensitivity and exceptional third-order nonlinearities ${ }^{13-16}$, making them a promising candidate for all optical processing and light written optical components ${ }^{17-21}$. In addition to novel optical properties, ChGs can be deposited at low temperatures $\left(<150^{\circ} \mathrm{C}\right)$ on numerous substrates through vapor or solution-based methods, which is in contrast with most crystalline material based waveguides that require epitaxial growth ${ }^{22,23}$. This has allowed us to integrate ChGs with unique substrate platforms such as flexible polymers and fluoride crystals ${ }^{24-28}$.

Recently we have shown that the ChG platform can also be utilized to enable direct photonic integration on 2D van der Waals (vdW) solids ${ }^{29-31}$. 2D van der Waals materials display a myriad of remarkable optical properties, such as tunable bandgaps in transition metal dichalcogenides (TMDCs), exceptional nonlinearities in III-VI compounds, and plasmonics in graphene $e^{32-35}$. However, their thickness prevents considerable light-matter interaction in the traditional surfacenormal-incident configurations. By placing the 2D materials on top planar waveguides or cavities, the light propagates parallel to the plane of the crystal and is no longer limited by its thickness ${ }^{36,37}$. Previous devices position $2 \mathrm{D}$ crystals on top of existing photonic structures, only utilizing the relatively weak evanescent fields and often necessitating additional planarization steps ${ }^{38-41}$. ChGs, like 2D materials, bond to their substrate through $\mathrm{vdW}$ interactions, allowing them to be easily deposited at room-temperature onto $2 \mathrm{D}$ crystals, serving both as a passivation layer and waveguide material. This glass-on-2D-material technology offers a promising route for rapid prototyping integrated photonics involving 2D materials. Most pristine 2D materials are still prepared by mechanical exfoliation, resulting in small micron-scale flakes. Through the glass-on-2D-material platform, the waveguides can be lithographically fabricated on top of the 2D sample, allowing for consideration of the flake's crystalline orientation and geometry. With an already large array of 2D materials, and an even larger number of potential stacked heterostructures, ChGs offer a convenient method to rapidly prototype 2D material based photonic devices from micron-scale crystals - in analogous to metal electrode patterning in 2D material electronic device prototyping.

*hujuejun@mit.edu

Smart Photonic and Optoelectronic Integrated Circuits XXII, edited by Sailing He, Laurent Vivien, Proc. of SPIE Vol. 11284, 112841U · (c) 2020 SPIE · CCC code: 0277-786X/20/\$21 · doi: 10.1117/12.2543619 
Tellurene, an emerging 2D vdW material which can be synthesized from solution with high throughput and quality, shows great promise for mid-IR integrated optoelectronics ${ }^{42,43}$. The synthesized free-standing tellurene crystals have large lateral dimensions (from tens to hundreds of microns) and process-controlled thickness (from a monolayer to tens of nanometers). All tellurene flakes grow laterally along the $\langle 0001\rangle$ and $\langle 1 \overline{2} 10\rangle$ directions, with the vertical stacking along the $\langle 10 \overline{1} 0\rangle$ directions. Tellurene offers several critical materials and processing advantages for mid-IR integrated photonics: 1) tellurene has a thickness-dependent bandgap $(\sim 0.35-1.2 \mathrm{eV})$ covering mid-IR bands up to $3.5 \mu \mathrm{m}$ wavelength $\left.{ }^{44,45} ; 2\right)$ it boasts excellent gating modulation on/off ratio $\left(\sim 10^{5}-10^{6}\right)$ and low carrier concentration after gating, a critical feature for reducing dark current in photodetectors and power consumption in our modulator design due to Joule heating; 3) tellurene is air-stable without encapsulation at ambient conditions; 4) tellurene's chain structure leads to the existence of the dangling bonds only at the (0001) ends of the molecular chains, thereby preventing recombination at the edges and ensuring long carrier lifetime; and 5) tellurene can be scalably produced via low-cost, substrate-free solution processing and assembled over large areas using a Langmuir-Blodgett (LB) process with predefined orientations, enabling the proposed devices and future explorations of tellurene on diverse designer substrates.

Tellurene synthesized following the solution route has recently been utilized to fabricate free-space photoconductive detectors with an already impressive room-temperature peak noise-equivalent power (NEP) of $0.28 \mathrm{pW} / \mathrm{Hz}^{1 / 2}$ at $1.7 \mu \mathrm{m}$ wavelength ${ }^{46}$. This initial demonstration corroborates the high optoelectronic quality of tellurene processed using the solution technique. In this work, we investigate waveguide integration of mid-IR tellurene detectors. In addition to the apparent advantage of facilitating on-chip integration, waveguide integration also introduces several important performance benefits. First, the longer path length made possible by waveguide integration contributes to enhanced optical absorption. This benefit is particularly pronounced if we are to extend the spectral range of the detector to the mid-IR $(2-3.6 \mu \mathrm{m})$, where response of the free space detector prototype significantly rolls off due to attenuated absorption ${ }^{46}$. Moreover, waveguide integration improves the signal-to-noise ratio (SNR). When light is funneled into the detector via a waveguide (with a core index $n$ ) rather than from free space, the detector active volume and hence noise can be reduced approximately by a factor of $n^{2}{ }^{47}$. Since most detector noises (Johnson, generation-recombination, and shot noises) scale with active volume ${ }^{48}$, the size scaling leads to suppressed noise. Finally, waveguide-integrated detectors can attain larger bandwidth than their free space counterparts thanks to their diminished RC delay and carrier transit time.

In addition to photodetectors, optical modulators and switches are vital components of a photonic circuit to enable signal switching and routing, data encoding, phase-sensitive detection, and spectroscopic interrogation ${ }^{49,50}$. Mid-IR modulators have attracted significant interests in recent years, and several modulator technologies have been developed in the midIR regime using thermo-optic phase shift ${ }^{51-53}$, free carrier plasma dispersion ${ }^{54,55}$, electro-absorption (Pauli blocking or field-induced effects) ${ }^{56-58}$, or electro-refractive (Pockels) effect ${ }^{59}$. Among them, Pockels electrooptic (EO) modulators are particularly attractive given their intrinsic ultrafast response $(>\mathrm{THz})$ and the capacity of achieving phase-only modulation - an important characteristic enabling scalable optical switching. To date, mid-IR integrated Pockels modulators have only been experimentally implemented in $\mathrm{Si}$-on- $\mathrm{LiNbO}_{3}{ }^{59}$ and theoretically analyzed in $\mathrm{AlN}$-on-SiO${ }_{2}{ }^{60}$. The designs mandate hybrid integration on less-standard substrates, operate over a limited mid-IR band $(<5 \mu \mathrm{m})$ bound by phonon absorption in the oxides, and demand high driving voltages ( $>50 \mathrm{~V})$ due to low EO activity in the materials.

2-D materials have demonstrated singular nonlinear optical properties ${ }^{61}$. In particular, the transition metal chalcogenides (TMDC) exhibit very strong second harmonic generation (large $\chi^{(2)}$ ), but only for few-layer thicknesses where the inversion symmetry of the crystal structure is broken ${ }^{62}$. This unfortunately limits their use for EO modulators, as the optical modal overlap with the electrooptic material would be too low for efficient modulation. Tellurene features extraordinary EO activity even as a bulk material, which makes it a superb candidate for mid-IR Pockels effect modulators. Bulk tellurium exhibits a large linear EO coefficient $d_{11}=9.2 \times 10^{-10} \mathrm{~m} / \mathrm{V}^{63}$. The refractive index change due to Pockels effect is given by:

$$
\Delta n_{E O}=-\frac{1}{2} n_{E O}{ }^{3} d_{11} E
$$

where $n_{E O}$ is the refractive index of the EO material (Te), $d_{11}$ is the EO tensor element, and $E$ is the applied electric field. In addition to its large EO coefficient, tellurene's high refractive index further enhances its EO activity according to Eq. 1: the EO activity of tellurene is 90 times larger than that of the gold standard $\mathrm{EO}$ material $\mathrm{LiNbO}_{3}$ !

Set aside its extraordinary EO activity, tellurene is also fundamentally different from traditional EO crystals in that it is a semiconductor rather than an electrical insulator, and thus motion of free carriers under applied bias significantly alters 
the device behavior. We note that this challenge is not unique to tellurene: strained $\mathrm{Si}$ is another semiconducting EO material potentially with tremendous technological importance. So far, EO devices utilizing strained Si have adopted an insulator-semiconductor-insulator (ISI) configuration (effectively two back-to-back MOS structures) ${ }^{64-66}$. In this configuration, the applied electric field is heavily screened by the free carriers in $\mathrm{Si}$, resulting in severely reduced Pockels effect. In fact, more recent studies have found that the earlier reports substantially overestimates the Pockels contribution from strained $\mathrm{Si}$ in these devices, and the observed second-order EO response mostly derives from free carrier and interface effects ${ }^{67-70}$. In this paper we propose a simple and effective design to counter the free carrier screening issue by operating the device in a resistive mode. In addition to achieving excellent performance with tellurene-based mid-IR modulators, the design can also utilize new semiconductor EO materials for optical modulation and switching over diverse spectral bands.

\section{ROOM-TEMPERATURE MID-IR TELLURENE PHOTODETECTOR}
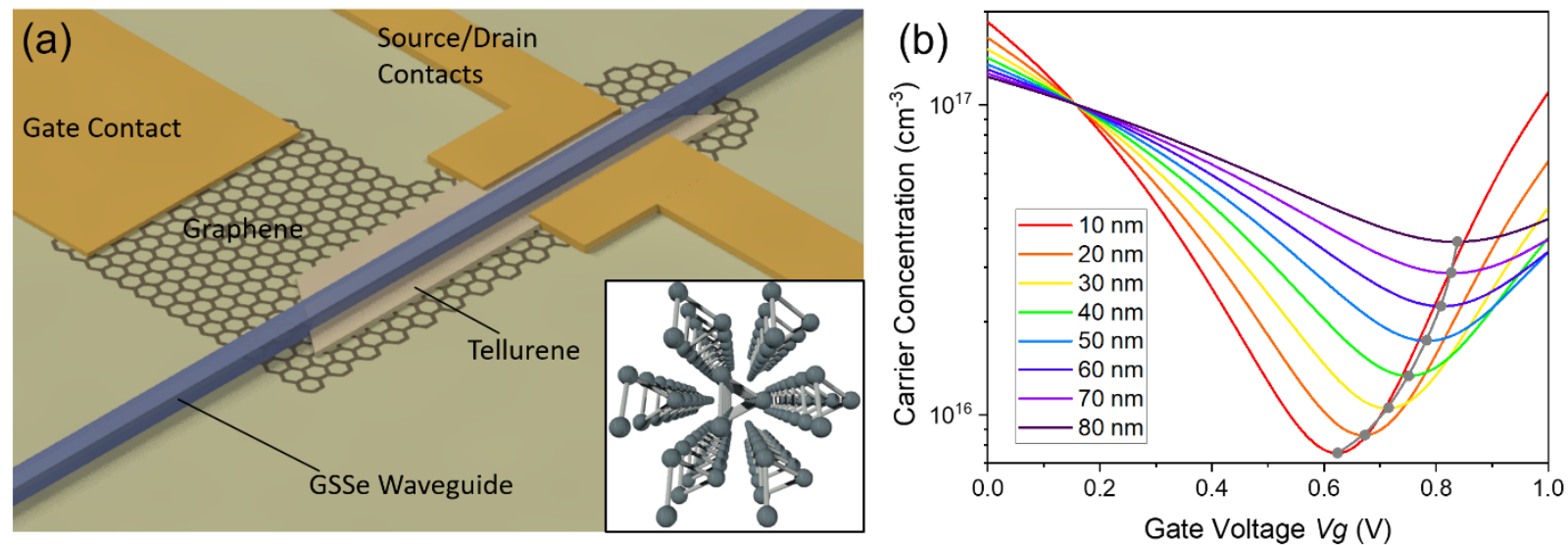

Figure 1. (a) Schematic of waveguide integrated tellurene photodetector. Inset shows view of tellurene crystal structure along [0001] axis. (b) Effective carrier concentration for different gate voltages in tellurene flakes of different thicknesses. The dotted gray line connects the minimum achievable carrier concentration for each thickness ${ }^{31}$.

Figure 1a depicts the waveguide-integrated photoconductive detector design consisting of a graphene bottom gate and a ChG waveguide on tellurene. The bottom gate is used to modulate the carrier concentration in Te to minimize dark current and also prohibit premature long wavelength cutoff due to Pauli blocking. The waveguide is aligned along the [0001] axis, a choice that contributes to extended operation spectral ranges given the in-plane optical absorption anisotropy of tellurene ${ }^{46}$. The detector is optimized to maximize its noise equivalent power (NEP), the figure-of-merit giving the signal-to-noise ratio of waveguide-integrated photodetectors ${ }^{71}$. Specifically, the design is based on these considerations: 1) the tellurene thickness is chosen such that it can be fully depleted with gating while maintaining sufficient modal confinement; 2) given the tellurene thickness, the waveguide dimensions are determined to ensure single-mode operation and minimize coupling loss into Te; 3 ) the detector length is optimized by considering the tradeoff between dark current (which linearly scales with detector length) and optical absorption; and 4) the metal contact spacing is chosen to balance responsivity and parasitic absorption. Minimum carrier concentrations in the gated tellurene were simulated by numerically solving the steady-state carrier distribution in the layer stack. Figure $1 \mathrm{~b}$ shows the minimum achievable carrier concentration in the graphene/ $\mathrm{Ge}_{23} \mathrm{Sb}_{7} \mathrm{~S}_{70}(20 \mathrm{~nm}) /$ tellurene metal-insulator-semiconductor (MIS) structure. A minimum carrier concentration of $7.5 \times 10^{15} \mathrm{~cm}^{-3}$ can be achieved for $10 \mathrm{~nm}$ thick tellurene. Thickness below $10 \mathrm{~nm}$ is undesirable for photodetectors, as the bandgap will begin to increase limiting the spectral range of the detector.

Figure 2a shows the side-view optical intensity distribution in a detector simulated using 3-D finite-difference timedomain (FDTD) based on measured material optical constants ${ }^{46}$. The design is optimized following the aforementioned procedures. Coupling into the tellurene absorber is highly efficient given the small tellurium thickness compared to the waveguide height $(700 \mathrm{~nm})$, which maintains an optical scattering loss at the flake edge below $0.1 \mathrm{~dB}$ from the modal mismatch (Figures 2b, 2c). Waveguide dimensions were chosen to allow single mode detection up to $3.5 \mu \mathrm{m}$. Using experimentally measured data of solution-synthesized tellurene (mobility along the [1210] axis: $240 \mathrm{~cm}^{2} \mathrm{~V}^{-1} \mathrm{~s}^{-1}$; carrier density: $10^{11} \mathrm{~cm}^{-2}$; photocarrier lifetime: $280 \mathrm{~ns}^{72}$ ), we can project the optimized NEP of the preliminary detector design 
to be $0.03 \mathrm{fW} / \mathrm{Hz}^{1 / 2}$ at $3 \mu \mathrm{m}$ wavelength. As can be seen from Figure $2 \mathrm{~d}$, this NEP value corresponds to an optimal device length to balance between sufficiently large optical absorption and a small detector length with reduced dark current. Furthermore, thinner tellurene allows for lower NEP because of its reduced dark current, despite its weaker absorption. The photodetector bandwidth is mainly limited by the carrier lifetime to be $0.57 \mathrm{MHz}$, comparable to other mid-IR photoconductive detectors ${ }^{73}$. Such performance, represents almost four orders of magnitude improvement in SNR compared to the free-space counterpart ${ }^{46}$. The NEP value is also far superior to the best figure previously reported for mid-IR waveguide-integrated detectors $\left(35 \mathrm{fW} / \mathrm{Hz}^{1 / 2}\right.$ in an InP-based multi-quantum-well detector hybrid bonded on $\mathrm{Si}^{74,75}$ ), while our device further claims the benefits of simple processing and fully monolithic integration on most substrates. The superior performance and Si-monolithic integration capacity qualify the tellurene device as a promising solution for mid-IR detection on-chip.
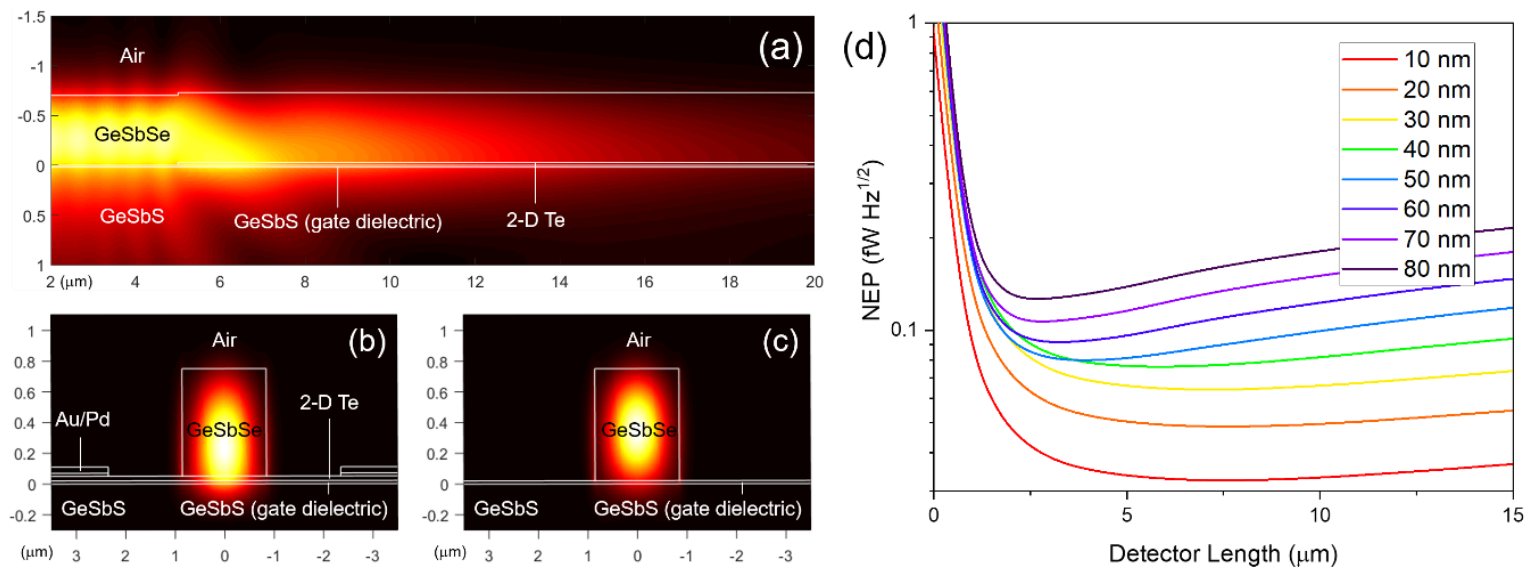

Figure 2. (a) Simulated optical intensity distribution in a waveguide-coupled tellurene mid-IR detector; (b, c) mode profiles inside the ChG waveguide (b) with and (c) without tellurene; (d) computed NEP of the detector as a function of the device length and tellurene thickness ${ }^{31}$.

\section{POCKELS EFFECT MID-IR MODULATOR}

Tellurene exhibits strong anisotropy along the two principle in-plane directions ([0001] and [1리]) due to its lowsymmetry lattice, a factor that must be properly considered in Pockels device design. To appropriately engineer a tellurene based EO modulator, we experimentally characterized anisotropy of tellurene's second-order nonlinear susceptibility tensor by measuring second harmonic generation (SHG) from solution-derived tellurene flakes using multiphoton microscopy. Figure $3 \mathrm{a}$ and $3 \mathrm{~b}$ show the reflected fundamental $(1300 \mathrm{~nm})$ and SHG $(650 \mathrm{~nm})$ of multiple tellurene flakes of different sizes and orientations. The tellurene crystals that have their [0001] direction vertical to the images (perpendicular to the fundamental polarization), demonstrate the most intense SHG. Tellurene's structure belongs to the $\mathrm{D}_{3}$ point group resulting in five non-vanishing second-order susceptibility tensor elements. Figure 3c shows the intensity of the second harmonic as a function of the relative angle between the tellurene flakes and the laser polarization. The cosine squared fit agrees with the $\mathrm{D}_{3}$ symmetry of tellurene. Knowing the orientation of the electric field relative to the tellurene's crystalline orientation, the change in the refractive index of the tellurene under the applied field can be calculated. Figure 3d shows how the refractive index of tellurene changes as the angle between the applied electric field and [0001] direction changes. The result indicates that the waveguide should align along the [0001] axis of tellurene, while the contacts adjacent to the waveguide will produce an electric field perpendicular to the [0001] direction to attain the maximum EO response.

Following the conclusion, the Pockels EO phase shifter layout is similar to that of the waveguide-integrated photodetector shown in Figure 1a. Instead of using an ISI structure, here the metal electrodes directly contact the tellurene. Voltage drop takes place almost entirely in tellurene, since the electrode contact resistance $(6 \Omega)$ is much smaller than tellurene's resistance ( $719 \Omega$ for $80 \mathrm{~nm}$ thick tellurene). The gate serves two purposes: by biasing the gate such that tellurene operates near the onset of inversion, the electrical resistance is maximized to increase the electric field in tellurene; additionally, electric current passing through tellurene and hence power consumption of the device is also minimized according to the Ohm's law. 

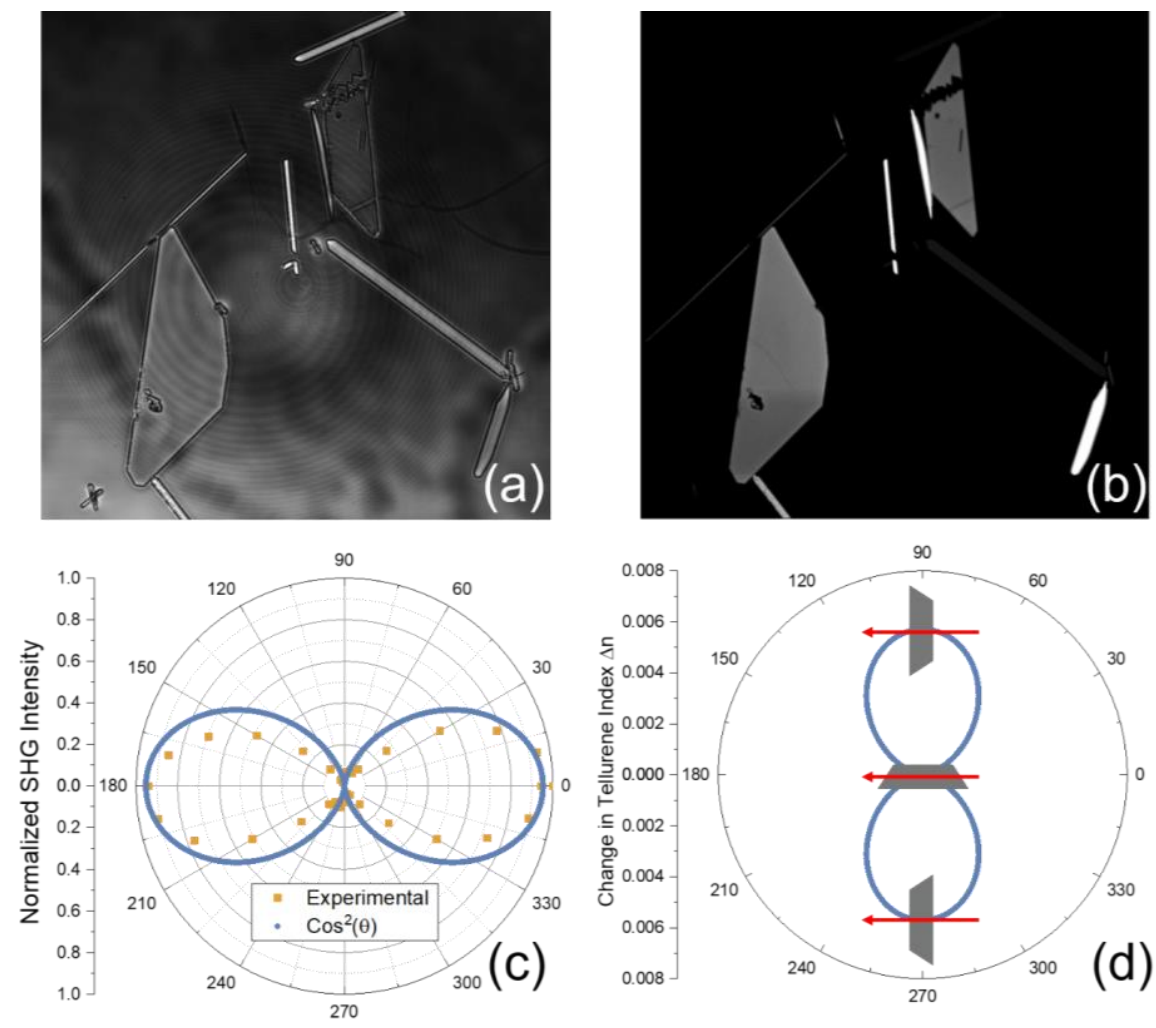

Figure 3. (a) Multiphoton microscope image of reflected $1300 \mathrm{~nm}$ fundamental beam (b) and $650 \mathrm{~nm}$ reflected SHG. Fundamental laser polarization is horizontal. (c) SHG intensity from tellurene samples as a function of incident laser polarization. The experimental data fit well to a cosine squared function in agreement with tellurium's crystal structure; (d) change in refractive index of tellurium as a function of the angle between the [0001] direction and an electric field of 103 $\mathrm{V} / \mathrm{cm}$. Insets show the orientation of tellurene (gray trapezoid) relative to electric field (red arrow) for the orientations corresponding to maximum and minimum change in refractive inde ${ }^{31}$.

The phase shifter design is optimized to minimize the half-wave voltage-length product $\left(V_{\pi} \cdot L\right)$ and power consumption. The product $V_{\pi} \cdot L$ is primarily dictated by the spatial overlap between the optical mode (at $4 \mu \mathrm{m}$ wavelength in our initial design) and the electric field in the EO medium. Figures $4 \mathrm{a}$ and $4 \mathrm{~b}$ present the simulated cross-sectional electric and optical field distribution in the EO phase shifter, showing large overlap between the fields in tellurene. The ChG waveguide size is chosen to be $2.5 \mu \mathrm{m} \times 0.71 \mu \mathrm{m}$ to maximize confinement factor (see Supporting Figure 4). Spatially dependent EO index modification $\Delta n_{E O}$ is then computed using Eq. 1, and the waveguide effective index modulation is derived following the classical perturbation theory. This approach allows us to quantify the $V_{\pi} \cdot L$ product for varying thickness of tellurene. According to the simulation results shown in Figure $4 \mathrm{c}, V_{\pi} \cdot L$ improves with increasing tellurene thickness benefiting from enhanced optical modal overlap, although the performance gain plateaus at larger tellurene thicknesses. At $40 \mathrm{~nm}$ tellurene thickness, the optimized $V_{\pi} \cdot L$ reaches $2.7 \mathrm{~V} \cdot \mathrm{cm}-$ representing 10 -fold improvement compared to state-of-the-art mid-IR EO modulators ${ }^{59}$. The superior $V_{\pi} \cdot L$ product of the EO phase shifter facilitates its integration in modulator devices with a compact footprint.

Unlike the traditional ISI configuration, where the phase shifter is a purely capacitive device (when leakage current is negligible), a distinctive feature of the new design is its resistive nature, similar to thermo-optic phase shifters. The phase shifter therefore continuously draws electrical power due to Joule heating. The power consumption of a modulator device based on the phase shifter design therefore encompasses both the continuous current flow and capacitive charging/discharging. We analyze the power consumption of a tellurene modulator assuming Mach-Zehnder interferometer (MZI, Figure 4d, inset) and racetrack resonator (Figure 4e, inset) configurations, both based on the phase shifter design. Figure $4 \mathrm{~d}$ and e plot the modulation energy-per-bit of the two configurations, respectively, for different thicknesses of tellurene. In the calculations, we account for the impact of modal overlap with tellurene, optical scattering loss at the tellurene waveguide interface, as well as the photon lifetime limit to modulation bandwidth. Resonators incorporating thinner flakes have higher Q-factors and lower photon-lifetime-limited bandwidth as a result of reduced 
scattering losses at the waveguide tellurene interfaces. The energy of the devices begins to rapidly increase beyond their photon-lifetime limited bandwidth due to the increased voltages necessary to drive the device at the same extinction ${ }^{76}$. Their higher Q-factors also lead to a smaller switching bias and lower energy per bit. Competing with both of these factors is the smaller modal overlap with the tellurene for thinner flakes resulting in a smaller $\mathrm{V}_{\pi} \cdot \mathrm{L}$. Ultimately, an optimized energy per bit of $12.0 \mathrm{pJ}$ at $70 \mathrm{GHz}$ modulation bandwidth can be achieved in $40 \mathrm{~nm}$ tellurene. This is attained in spite of the unfavorable $\lambda^{2}$ (wavelength squared) scaling of the $\mathrm{V}_{\pi} \cdot \mathrm{L}$ product for EO modulators. The result indicates that the resistive EO modulator design can overcome the free carrier screening issue while offering competitive performance in terms of energy consumption.
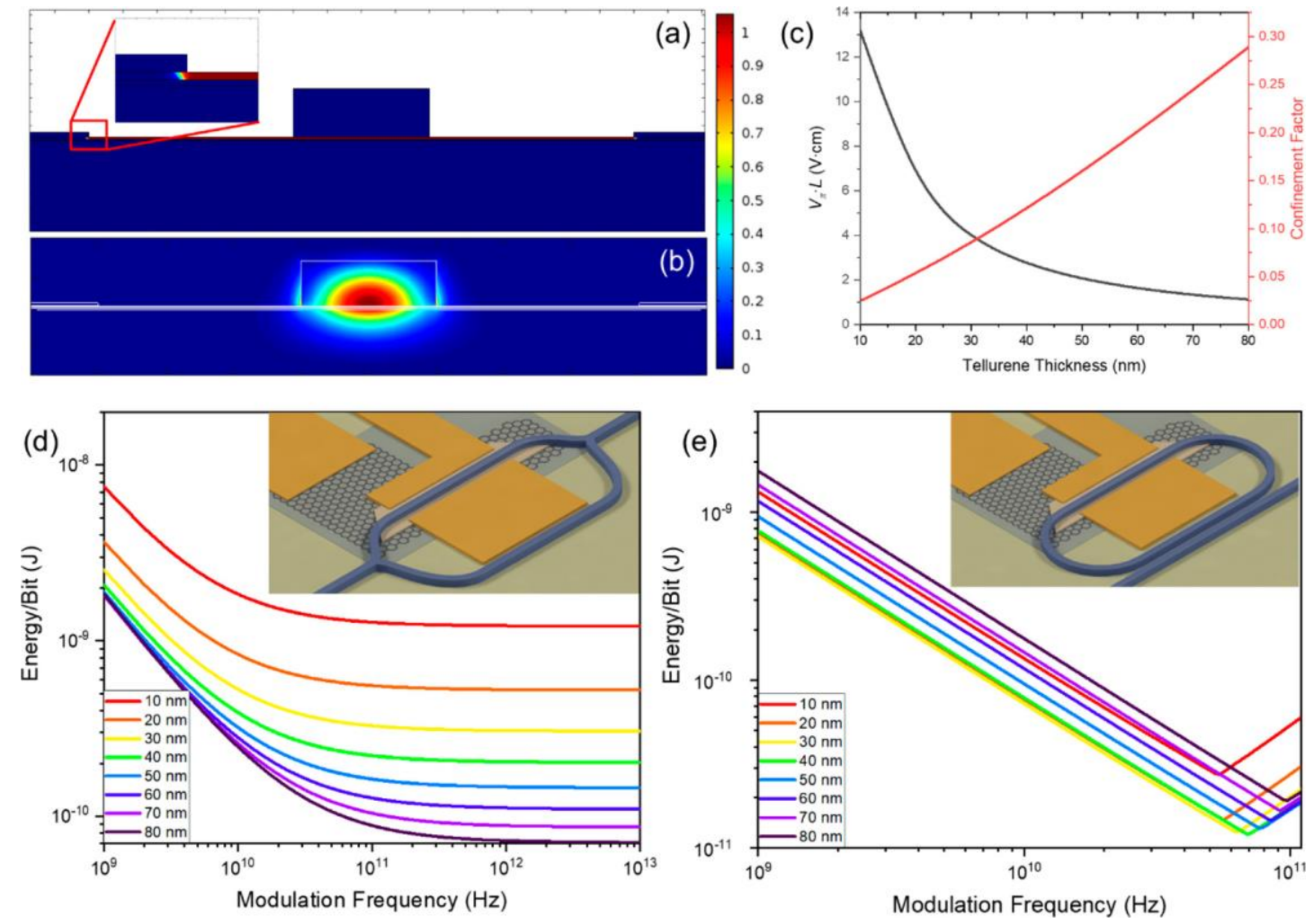

Figure 4. (a, b) Simulated cross-sectional (a) electric and (b) optical field profiles in the modulator. (c) Simulated confinement factor and voltagelength product for different tellurene thicknesses. (d) Simulated energy consumption of the modulator vs frequency for different 2-D Te thicknesses in MZI architecture tellurene modulator. Inset shows schematic of MZI modulator design (e) Simulated energy consumption of tellurene racetrack modulator vs frequency for different tellurene thickness. Inset shows schematic of racetrack modulator design ${ }^{31}$.

\section{CONCLUSION}

This work demonstrates the promise of tellurene as a multifunctional material for integrated optoelectronic devices in the mid-IR. Tellurene's small bandgap and low gated carrier concentration enable extremely low noise photodetector at room temperature. Additionally, tellurene's broken structural inversion symmetry and giant electrooptic activity allow it to be employed for extremely fast and low energy Pockels effect modulators. Both detector and modulator devices achieve performances significantly better compared to existing state-of-the-art. The capability of scalable synthesis and assembly of tellurene on arbitrary substrates at low temperatures further facilitates its integration with functional integrated photonic circuits. We therefore foresee that tellurene will become a powerful and versatile material platform for mid-IR optoelectronic applications.

\section{ACKNOWLEDGEMENTS}

This material is based upon work supported by the National Science Foundation under award numbers 1453218 and 1506605, Graduate Research Fellowship under Grant No. 1122374. The authors also acknowledge facility support by the Harvard University Center for Nanoscale Systems, the latter of which is supported by the National Science Foundation under award 0335765. 


\section{REFERENCES}

[1] Hu, J., Li, L., Lin, H., Zou, Y., Du, Q., Smith, C., Novak, S., Richardson, K., and Musgraves, J.D., "Chalcogenide glass microphotonics: Stepping into the spotlight," American Ceramic Society Bulletin 94(4), 24-29 (2015).

[2] Eggleton, B.J.J., Luther-Davies, B., and Richardson, K., "Chalcogenide photonics," Nature Photonics 5(3), 141148 (2011).

[3] Vigreux-Bercovici, C., Bonhomme, E., and Pradel, A., "Te-rich Ge-As-Se-Te bulk glasses and films for future IR-integrated optics," Journal of Non-Crystalline Solids 353(13-15 SPEC. ISS.), 1388-1391 (2007).

[4] Zou, Y., Zhang, D., Lin, H., Li, L., Moreel, L., Zhou, J., Du, Q., Ogbuu, O., Danto, S., et al., "HighPerformance, High-Index-Contrast Chalcogenide Glass Photonics on Silicon and Unconventional Non-planar Substrates," Advanced Optical Materials 2, 478-486 (2014).

[5] Du, Q., Huang, Y., Li, J., Kita, D., Michon, J., Lin, H., Li, L., Novak, S., Richardson, K., et al., "Low-loss photonic device in $\mathrm{Ge}-\mathrm{Sb}-\mathrm{S}$ chalcogenide glass," Optics letters 41(13), 3090-3093 (2016).

[6] Madden, S.J., Choi, D.-Y., Bulla, D.A., Rode, A. V, Luther-Davies, B., Ta'eed, V.G., Pelusi, M.D., and Eggleton, B.J., "Long, low loss etched As2S3 chalcogenide waveguides for all-optical signal regeneration.," Optics Express 15(22), 14414-21 (2007).

[7] Geiger, S., Du, Q., Huang, B., Shalaginov, M.Y., Michon, J., Lin, H., Gu, T., Yadav, A., Richardson, K.A., et al., "Understanding aging in chalcogenide glass thin films using precision resonant cavity refractometry," Optical Materials Express 9(5), 2252 (2019).

[8] Lucas, P., Yang, Z., Fah, M.K., Luo, T., Jiang, S., Boussard-Pledel, C., Anne, M.-L., and Bureau, B., "Telluride glasses for far infrared photonic applications," Optical Materials Express 3(8), 1049 (2013).

[9] Le Coq, D., Cui, S., Boussard-Plédel, C., Masselin, P., Bychkov, E., and Bureau, B., "Telluride glasses with farinfrared transmission up to $35 \mu \mathrm{m}$," Optical Materials 72, 809-812 (2017).

[10] Lin, H., Li, L., Deng, F., Ni, C., Danto, S., Musgraves, J.D., Richardson, K., and Hu, J., "Demonstration of midinfrared waveguide photonic crystal cavities," Optics Letters 38(15), 2779-2782 (2013).

[11] Lin, H., Li, L., Zou, Y., Danto, S., Musgraves, J.D., Richardson, K., Kozacik, S., Murakowski, M., Prather, D., et al., "Demonstration of high-Q mid-infrared chalcogenide glass-on-silicon resonators," Optics Letters 38(9), 1470 (2013).

[12] Du, Q., Luo, Z., Zhong, H., Zhang, Y., Huang, Y., Du, T., Zhang, W., Gu, T., and Hu, J., "Chip-scale broadband spectroscopic chemical sensing using an integrated supercontinuum source in a chalcogenide glass waveguide," Photonics Research 6(6), 506 (2018).

[13] Ta 'eed, V.G., Baker, N.J., Fu, L., Finsterbusch, K., Lamont, M.R.E., Moss, D.J., Nguyen, H.C., Eggleton, B.J., Choi, D.Y., et al., "Ultrafast all-optical chalcogenide glass photonic circuits," Optics express 15(15), 9205-9221 (2007).

[14] Wang, T., Gai, X., Wei, W., Wang, R., Yang, Z., Shen, X., Madden, S., and Luther-Davies, B., "Systematic zscan measurements of the third order nonlinearity of chalcogenide glasses," Optical Materials Express 4(5), 1011 (2014).

[15] Almeida, J.M.P., Barbano, E.C., Arnold, C.B., Misoguti, L., and Mendonça, C.R., "Nonlinear optical waveguides in As_2S_3-Ag_2S chalcogenide glass thin films," Optical Materials Express 7(1), 93 (2017).

[16] Yu, Y., Gai, X., Ma, P., Choi, D.Y., Yang, Z., Wang, R., Debbarma, S., Madden, S.J., and Luther-Davies, B., "A broadband, quasi-continuous, mid-infrared supercontinuum generated in a chalcogenide glass waveguide," Laser and Photonics Reviews 8(5), 792-798 (2014).

[17] Hughes, M., Yang, W., and Hewak, D., "Fabrication and characterization of femtosecond laser written waveguides in chalcogenide glass," Applied Physics Letters 90(13), 131113-42008 (2007).

[18] Mairaj, A.K., Riziotis, C., Chardon, A.M., Smith, P.G.R., Shepherd, D.P., and Hewak, D.W., "Development of channel waveguide lasers in Nd3+-doped chalcogenide (Ga:La:S) glass through photoinduced material modification," Applied Physics Letters 81(20), 3708-3710 (2002).

[19] Lee, M.W., Grillet, C., Smith, C.L.C., Moss, D.J., Eggleton, B.J., Freeman, D., Luther-Davies, B., Madden, S., Rode, A., et al., "Photosensitive post tuning of chalcogenide photonic crystal waveguides.," Optics express 15(3), 1277-85 (2007). 
[20] Musgraves, J.D., Richardson, K., and Jain, H., "Laser-induced structural modification, its mechanisms, and applications in glassy optical materials," Optical Materials Express 1(5), 921 (2011).

[21] Hu, J., Torregiani, M., Morichetti, F., Carlie, N., Agarwal, A., Richardson, K., Kimerling, L.C., and Melloni, A., "Resonant cavity-enhanced photosensitivity in As2S3 chalcogenide glass at $1550 \mathrm{~nm}$ telecommunication wavelength.," Optics letters 35(6), 874-876 (2010).

[22] Zha, Y., Waldmann, M., and Arnold, C.B., "A review on solution processing of chalcogenide glasses for optical components," Optical Materials Express 3(9), 1259 (2013).

[23] Musgraves, J.D., Carlie, N., Hu, J., Petit, L., Agarwal, A., Kimerling, L.C., and Richardson, K.A., "Comparison of the optical, thermal and structural properties of Ge-Sb-S thin films deposited using thermal evaporation and pulsed laser deposition techniques," Acta Materialia 59(12), 5032-5039 (2011).

[24] Li, L., Lin, H., Qiao, S., Zou, Y., Danto, S., Richardson, K., Musgraves, J.D., Lu, N., and Hu, J., "Integrated flexible chalcogenide glass photonic devices," Nature Photonics 8(8), 643-649 (2014).

[25] Hu, J., Li, L., Lin, H., Zhang, P., Zhou, W., and Ma, Z., "Flexible integrated photonics: where materials, mechanics and optics meet [Invited]," Optical Materials Express 3, 1313-1331 (2013).

[26] Zha, Y., Lin, P.T., Kimerling, L., Agarwal, A., and Arnold, C.B., "Inverted-Rib Chalcogenide Waveguides by Solution Process," ACS Photonics 1(3), 153-157 (2014).

[27] Abdel-Moneim, N.S., Mellor, C.J., Benson, T.M., Furniss, D., and Seddon, A.B., "Fabrication of stable, low optical loss rib-waveguides via embossing of sputtered chalcogenide glass-film on glass-chip," Optical and Quantum Electronics 47(2), 351-361 (2014).

[28] Li, L., Lin, H., Qiao, S., Huang, Y.-Z., Li, J.-Y., Michon, J., Gu, T., Alosno-Ramos, C., Vivien, L., et al., "Monolithically integrated stretchable photonics," Light: Science \& Applications 7(2), 17138 (2018).

[29] Lin, H., Song, Y., Huang, Y., Kita, D., Deckoff-Jones, S., Wang, K., Li, L., Li, J., Zheng, H., et al., "Chalcogenide glass-on-graphene photonics," Nature Photonics 11(12), 798-805 (2017).

[30] Deckoff-Jones, S., Lin, H., Kita, D., Zheng, H., Li, D., Zhang, W., and Hu, J., "Chalcogenide glass waveguideintegrated black phosphorus mid-infrared photodetectors," Journal of Optics 20(4), 044004 (2018).

[31] Deckoff-Jones, S., Wang, Y., Lin, H., Wu, W., and Hu, J., "Tellurene: A Multifunctional Material for Midinfrared Optoelectronics," ACS Photonics 6(7), 1632-1638 (2019).

[32] Wang, Q.H., Kalantar-Zadeh, K., Kis, A., Coleman, J.N., and Strano, M.S., "Electronics and optoelectronics of two-dimensional transition metal dichalcogenides," Nature Nanotechnology 7(11), 699-712 (2012).

[33] Bonaccorso, F., Sun, Z., Hasan, T., and Ferrari, A.C., "Graphene photonics and optoelectronics," Nature Photonics 4(9), 611-622 (2010).

[34] Zhou, X., Cheng, J., Zhou, Y., Cao, T., Hong, H., Liao, Z., Wu, S., Peng, H., Liu, K., et al., "Strong SecondHarmonic Generation in Atomic Layered GaSe," Journal of the American Chemical Society 137(25), 7994-7997 (2015).

[35] Deckoff-Jones, S., Zhang, J., Petoukhoff, C.E., Man, M.K.L., Lei, S., Vajtai, R., Ajayan, P.M., Talbayev, D., Madéo, J., et al., "Observing the interplay between surface and bulk optical nonlinearities in thin van der Waals crystals," Scientific Reports 6, 22620 (2016).

[36] Youngblood, N., and Li, M., "Integration of 2D materials on a silicon photonics platform for optoelectronics applications," Nanophotonics 6(6), 1205-1218 (2017).

[37] Xia, F., Wang, H., Xiao, D., Dubey, M., and Ramasubramaniam, A., "Two-dimensional material nanophotonics," Nature Photonics 8(12), 899-907 (2014).

[38] Hu, Y.T., Pantouvaki, M., Brems, S., Asselberghs, I., Huyghebaert, C., Geisler, M., Alessandri, C., Baets, R., Absil, P., et al., "Broadband 10Gb/s graphene electro-absorption modulator on silicon for chip-level optical interconnects," 2014 IEEE International Electron Devices Meeting(c), 5.6.1-5.6.4 (2014).

[39] Schall, D., Neumaier, D., Mohsin, M., Chmielak, B., Bolten, J., Porschatis, C., Prinzen, A., Matheisen, C., Kuebart, W., et al., "50 GBit/s Photodetectors Based on Wafer-Scale Graphene for Integrated Silicon Photonic Communication Systems," ACS Photonics 1(9), 781-784 (2014).

[40] Gan, X., Shiue, R.J., Gao, Y., Meric, I., Heinz, T.F., Shepard, K., Hone, J., Assefa, S., and Englund, D., “Chipintegrated ultrafast graphene photodetector with high responsivity," Nature Photonics 7, 883-887 (2013).

[41] Li, H., Anugrah, Y., Koester, S.J., and Li, M., "Optical absorption in graphene integrated on silicon waveguides Dyadic Green's functions and guided surface waves for a surface conductivity model of graphene," Citation: Appl. Phys. Lett. Journal of Applied Physics Applied Physics Letters Journal of Applied Physics 101(117), 111110-171107 (2012). 
[42] Wang, Y., Qiu, G., Wang, R., Huang, S., Wang, Q., Liu, Y., Du, Y., Goddard, W.A., Kim, M.J., et al., "Fieldeffect transistors made from solution-grown two-dimensional tellurene," Nature Electronics 1(4), 228-236 (2018).

[43] Huang, S., Segovia, M., Yang, X., Koh, Y.R., Wang, Y., Ye, P.D., Wu, W., Shakouri, A., Ruan, X., et al., "Anisotropic thermal conductivity in 2D tellurium," 2D Materials 7(1), 15008 (2019).

[44] Zhu, Z., Cai, X., Yi, S., Chen, J., Dai, Y., Niu, C., Guo, Z., Xie, M., Liu, F., et al., "Multivalency-Driven Formation of Te-Based Monolayer Materials: A Combined First-Principles and Experimental study," Physical Review Letters 119(10), 106101 (2017).

[45] Coker, A., Lee, T., and Das, T.P., "Investigation of the electronic properties of tellurium—energy-band structure," Physical Review B 22(6), 2968-2975 (1980).

[46] Amani, M., Tan, C., Zhang, G., Zhao, C., Bullock, J., Song, X., Kim, H., Shrestha, V.R., Gao, Y., et al., "Solution-Synthesized High-Mobility Tellurium Nanoflakes for Short-Wave Infrared Photodetectors," ACS Nano 12(7), 7253-7263 (2018).

[47] Lin, H., Luo, Z., Gu, T., Kimerling, L.C., Wada, K., Agarwal, A., and Hu, J., "Mid-infrared integrated photonics on silicon: a perspective," Nanophotonics 7(2), 393-420 (2017).

[48] Dereniak, E.L., and Boreman, G.D. (Glenn D.., [Infrared detectors and systems], Wiley (1996).

[49] Kita, D.M., Lin, H., Agarwal, A., Richardson, K., Luzinov, I., Gu, T., and Hu, J., "On-Chip Infrared Spectroscopic Sensing: Redefining the Benefits of Scaling," IEEE Journal of Selected Topics in Quantum Electronics 23(2), 340-349 (2017).

[50] Kita, D.M., Miranda, B., Favela, D., Bono, D., Michon, J., Lin, H., Gu, T., and Hu, J., "High-performance and scalable on-chip digital Fourier transform spectroscopy," Nature Communications 9(1), 4405 (2018).

[51] Zou, Y., Chakravarty, S., Chung, C.-J., and Chen, R.T., "Miniature mid-infrared thermooptic switch with photonic crystal waveguide based silicon-on-sapphire Mach-Zehnder interferometers," 4 April 2016, 97530Q.

[52] Malik, A., Dwivedi, S., Van Landschoot, L., Muneeb, M., Shimura, Y., Lepage, G., Van Campenhout, J., Vanherle, W., Van Opstal, T., et al., "Ge-on-Si and Ge-on-SOI thermo-optic phase shifters for the mid-infrared," Optics Express 22(23), 28479 (2014).

[53] Nedeljkovic, M., Stankovic, S., Mitchell, C.J., Khokhar, A.Z., Reynolds, S.A., Thomson, D.J., Gardes, F.Y., Littlejohns, C.G., Reed, G.T., et al., "Mid-Infrared Thermo-Optic Modulators in SoI," IEEE Photonics Technology Letters 26(13), 1352-1355 (2014).

[54] Shen, L., Healy, N., Mitchell, C.J., Penades, J.S., Nedeljkovic, M., Mashanovich, G.Z., and Peacock, A.C., "Mid-infrared all-optical modulation in low-loss germanium-on-silicon waveguides," Optics Letters 40(2), 268 (2015).

[55] Van Camp, M.A., Assefa, S., Gill, D.M., Barwicz, T., Shank, S.M., Rice, P.M., Topuria, T., and Green, W.M.J., "Demonstration of electrooptic modulation at 2165nm using a silicon Mach-Zehnder interferometer," Optics Express 20(27), 28009 (2012).

[56] Yao, Y., Shankar, R., Kats, M.A., Song, Y., Kong, J., Loncar, M., and Capasso, F., "Electrically Tunable Metasurface Perfect Absorbers for Ultrathin Mid-Infrared Optical Modulators,” Nano Letters 14(11), 6526-6532 (2014).

[57] Peng, R., Khaliji, K., Youngblood, N., Grassi, R., Low, T., and Li, M., "Midinfrared Electro-optic Modulation in Few-Layer Black Phosphorus," Nano Letters 17(10), 6315-6320 (2017).

[58] Whitney, W.S., Sherrott, M.C., Jariwala, D., Lin, W.-H., Bechtel, H.A., Rossman, G.R., and Atwater, H.A., "Field Effect Optoelectronic Modulation of Quantum-Confined Carriers in Black Phosphorus," Nano Letters 17(1), 78-84 (2017).

[59] Chiles, J., and Fathpour, S., "Mid-infrared integrated waveguide modulators based on silicon-on-lithium-niobate photonics," Optica 1(5), 350 (2014).

[60] Liu, S., Xu, K., Song, Q., Cheng, Z., and Tsang, H., "Design of Mid-infrared electro-optic modulators based on aluminum nitride waveguides," Journal of Lightwave Technology1-1 (2016).

[61] Autere, A., Jussila, H., Dai, Y., Wang, Y., Lipsanen, H., and Sun, Z., "Nonlinear Optics with 2D Layered Materials," in Adv. Mater., Wiley-Blackwell, p. 1705963 (2018).

[62] Li, Y., Rao, Y., Mak, K.F., You, Y., Wang, S., Dean, C.R., and Heinz, T.F., "Probing symmetry properties of few-layer MoS2 and h-BN by optical second-harmonic generation," Nano Letters 13(7), 3329-3333 (2013).

[63] Jerphagnon, J., “Contribution a L'étude de Certains Phénomènes D’optique Non Linéaire,” Annales Des Télécommunications 23(7-8), 203-232 (1968). 
[64] Chmielak, B., Waldow, M., Matheisen, C., Ripperda, C., Bolten, J., Wahlbrink, T., Nagel, M., Merget, F., and Kurz, H., "Pockels effect based fully integrated, strained silicon electro-optic modulator," Optics Express 19(18), 17212 (2011).

[65] Jacobsen, R.S., Andersen, K.N., Borel, P.I., Fage-Pedersen, J., Frandsen, L.H., Hansen, O., Kristensen, M., Lavrinenko, A. V., Moulin, G., et al., "Strained silicon as a new electro-optic material," Nature 441(7090), 199202 (2006).

[66] Damas, P., Le Roux, X., Le Bourdais, D., Cassan, E., Marris-Morini, D., Izard, N., Maroutian, T., Lecoeur, P., and Vivien, L., "Wavelength dependence of Pockels effect in strained silicon waveguides," Optics Express 22(18), 22095 (2014).

[67] Cazzanelli, M., and Schilling, J., "Second order optical nonlinearity in silicon by symmetry breaking," Applied Physics Reviews 3(1), 011104 (2016).

[68] Borghi, M., Mancinelli, M., Merget, F., Witzens, J., Bernard, M., Ghulinyan, M., Pucker, G., and Pavesi, L., "High-frequency electro-optic measurement of strained silicon racetrack resonators," Optics Letters 40(22), 5287 (2015).

[69] Sharif Azadeh, S., Merget, F., Nezhad, M.P., and Witzens, J., "On the measurement of the Pockels effect in strained silicon," Optics Letters 40(8), 1877 (2015).

[70] Schriever, C., Bianco, F., Cazzanelli, M., Ghulinyan, M., Eisenschmidt, C., de Boor, J., Schmid, A., Heitmann, J., Pavesi, L., et al., "Second-Order Optical Nonlinearity in Silicon Waveguides: Inhomogeneous Stress and Interfaces," Advanced Optical Materials 3(1), 129-136 (2015).

[71] Li, L., Lin, H., Huang, Y., Shiue, R.-J., Yadav, A., Li, J., Michon, J., Englund, D., Richardson, K., et al., "Highperformance flexible waveguide-integrated photodetectors," Optica 5(1), 44 (2018).

[72] Guthmann, C., Hermann, C., and Thuillier, J.M., "Recombination phenomena in tellurium," Physica Status Solidi (a) 3(2), 365-374 (1970).

[73] Capper, P., and Elliott, C.T., Eds., [Infrared Detectors and Emitters: Materials and Devices] , Springer US, Boston, MA (2001).

[74] Wang, R., Muneeb, M., Sprengel, S., Boehm, G., Malik, A., Baets, R., Amann, M.-C., and Roelkens, G., "III-Von-silicon 2- $\mu \mathrm{m}$-wavelength-range wavelength demultiplexers with heterogeneously integrated InP-based type-II photodetectors," Optics Express 24(8), 8480 (2016).

[75] Wang, R., Sprengel, S., Muneeb, M., Boehm, G., Baets, R., Amann, M.-C., and Roelkens, G., " 2 m wavelength range InP-based type-II quantum well photodiodes heterogeneously integrated on silicon photonic integrated circuits," Optics Express 23(20), 26834 (2015).

[76] Lin, H., Ogbuu, O., Liu, J., Zhang, L., Michel, J., and Hu, J., "Breaking the Energy-Bandwidth Limit of Electrooptic Modulators: Theory and a Device Proposal,” Journal of Lightwave Technology 31(24), 4029-4036 (2013). 